\title{
The Hans algorithm failed to predict outcome in patients with diffuse large B-cell lymphoma treated with rituximab
}

\author{
K. BENESOVA ${ }^{1, *}$, K. FORSTEROVA ${ }^{1}$, H. VOTAVOVA ${ }^{1}$, V. CAMPR ${ }^{2}$, J. STRITESKY ${ }^{3}$, Z. VELENSKA ${ }^{3}$, B. PROCHAZKA ${ }^{4}$, R. PYTLIK ${ }^{1}$, M. TRNENY ${ }^{1}$ \\ ${ }^{1} 1$ st Department of Medicine- Department of Hematology, General Teaching Hospital and Charles University in Prague, 1st Faculty of Medicine; \\ ${ }^{2}$ Department of Pathology and Molecular Medicine, Charles University in Prague, 2 nd Faculty of Medicine; ${ }^{3}$ Department of Pathology, General \\ Teaching Hospital and Charles University in Prague, 1st Faculty of Medicine; ${ }^{4}$ Department of Biostatistics, The National Institute of Public \\ Health, Prague
}

*Correspondence: katerina.benesova@hotmail.com

Received February 27, 2012 / Accepted July 9, 2012

\begin{abstract}
Diffuse large B-cell lymphoma (DLBCL) consists of at least two biologically and pathogenetically different subtypes, the germinal centre B-cell (GCB) and the activated B cell type (ABC). It has been suggested that immunohistochemistry can discriminate these subtypes as well. The aim of this study was to verify the validity of the most commonly used Hans algorithm in patients with DLBCL treated with anthracycline- based chemotherapy with rituximab. Immunohistochemical staining using standard protocols was performed on formalin fixed paraffin-embedded tissues. CD20, CD5, CD23, BCL2, CD10, BCL6, MUM1 and Ki67 antibodies were applied. Out of 120 examined cases 52 patients were evaluated as GCB type and 68 patients as having non-GCB, out of a set of 99 patients treated with immunochemotherapy 45 patients with GCB and 54 patients with non-GCB DLBCL were identified. In this set of patients, there was no statistically significant difference neither in overall survival (OS) (HR 1.47 95\% CI 0.51-2.63; p=0.45) nor in progression free survival (PFS) (HR 1.57, $95 \%$ CI 0.76-3.22; $\mathrm{p}=0.731$ ) between both groups.
\end{abstract}

Key words: DLBCL, Hans, rituximab, GC, nonGC

Diffuse large B-cell lymphoma represents the most frequent lymphoma subtype, involving some $30-45 \%$ of all lymphomas in the USA and Europe[1,2] . Overall survival of patients treated with immuno- chemotherapy ranges from 40 to $90 \%$. The variability of this interval is due to a number of clinical and morphological prognostic factors. Based on gene expression profiling (GEP), DLBCL can be divided into germinal centre B-cell (GCB) lymphoma, activated B-cell (ABC) lymphoma and type 3 (T3) - unspecified lymphoma [3]. It has been demonstrated that this classification also has a prognostic significance, which is retained even if immunochemotherapy is used [4]. As the GEP is financially and technically demanding, requiring native frozen tissue processed in highly specialized laboratories, immunohistochemical methods have been developed to distinguish these two subtypes. The most frequently used method is the Hans algorithm [5]. In our work, we tried to verify the validity of this method in routine clinical practice using anthracyclinebased immunochemotherapy.

\section{Patients and methods}

Patients. We selected a total of 127 patients with de novo DLBCL diagnosed at the Institute of Pathology of the General Teaching Hospital or at the Institute of Pathology and Molecular Medicine of Motol Teaching Hospital and the $2^{\text {nd }}$ Faculty of Medicine. Seven patients were excluded from the cohort as the interpretation of the immunohistochemical findings in limited diagnostic material was not unequivocal. A total of 120 patients were thus examined according to Hans algorithm.

Basic clinical data were completed in all patients. Patients signed an informed consent regarding the processing of clinical data. The following data were available: date of diagnosis, age, sex, clinical stage (CS), performance status (PS), international prognostic index (IPI), lactate dehydrogenase (LDH) value, number of extranodal sites involved, type of chemotherapy and type of monoclonal antibody, quality of response, date of first progression, date of death and date of last follow-up. 


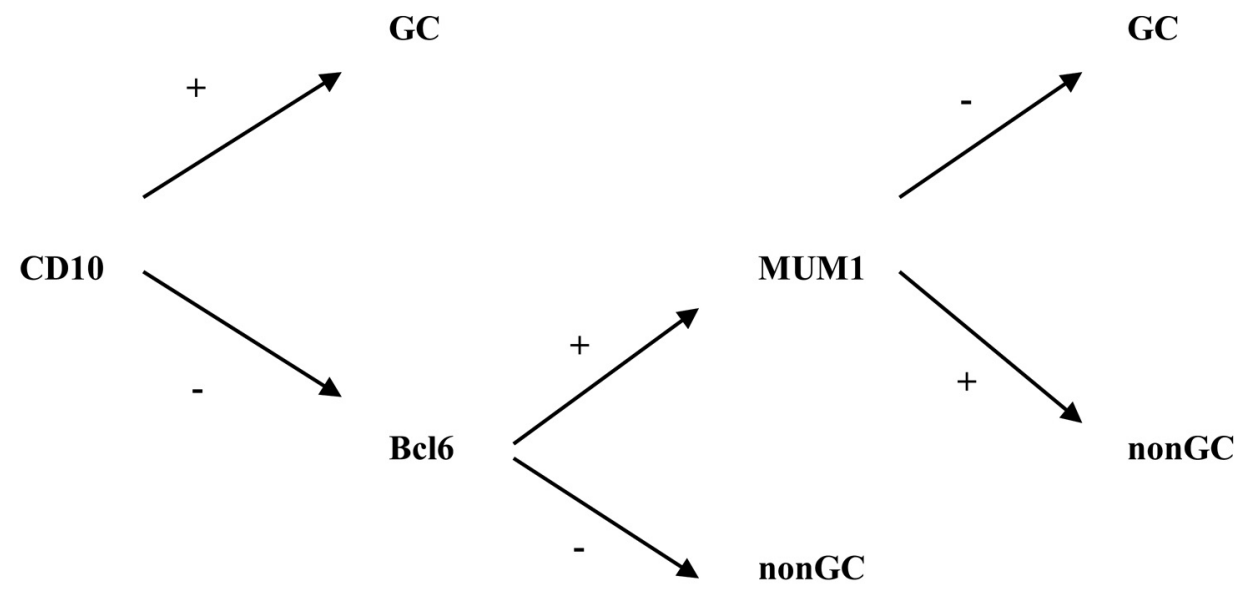

Figure 1a. The scheme of Hans algorithm

A
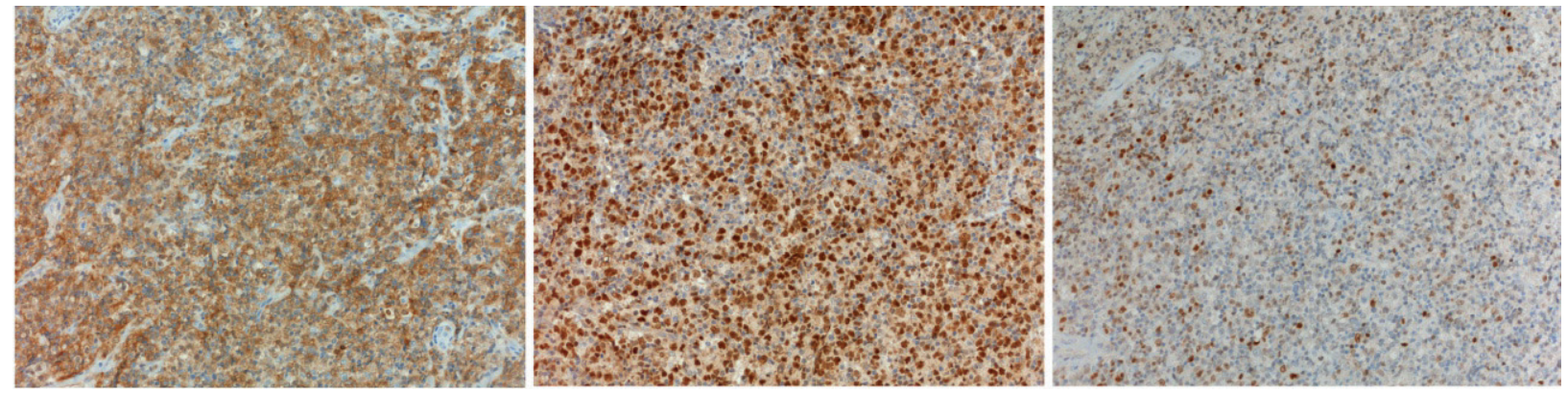

CD10

BCL-6

MUM1

B
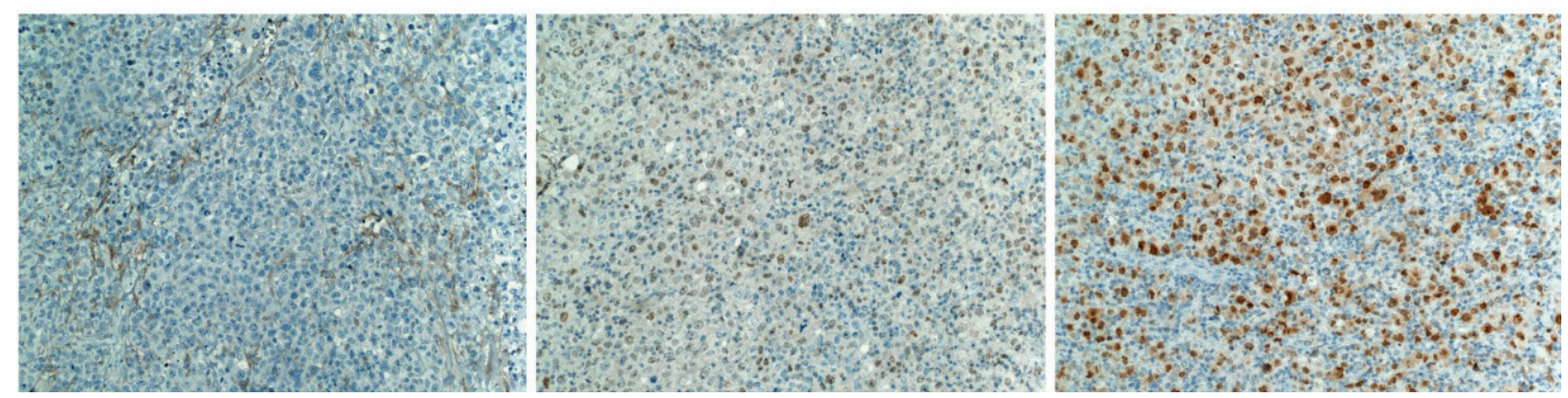

Figure 1b. Results of immunoperoxidase staining.

A: Diffuse large B-cell lymphoma with GC-like immunophenotype. CD10: Variable, moderate to strong membrane positivity of CD10 on tumour cells. BCL-6: Variable, often strong nuclear positivity of BCL6 on tumour cells. MUM1: Variable, often weak nuclear positivity of MUM1 on less than $30 \%$ of tumour cells.

B: Diffuse large B-cell lymphoma with non-GC-like immunophenotype. CD10: Stromal staining of CD10, tumour cells is negative.

The patients with unavailable sufficient number of basic clinical data, follow up or not treated with immunochemotherapy (rituximab, anthracycline-based chemotherapy) were excluded from the survival analysis. The remaining cohort consisted out of 99 patients diagnosed between 2001-2010. Response rate was evaluated using the Cheson criteria [6], due to fact that only limited number of patients were examined by PET.
Treatment. Patients were treated with the R-CHOP regimen (rituximab, cyclophosphamide, adriablastine, vincristine, prednisone) at standard dosage or in intensified version ${ }^{7}$.

Immunohistochemistry (IHC). Routinely prepared formalin-fixed paraffin-embedded (FFPE) tissue blocks were used for the diagnosis of diffuse large B-cell lymphoma according to the WHO classification criteria. Two pathologists (V.C., J.S.) independently read the slides to confirm 


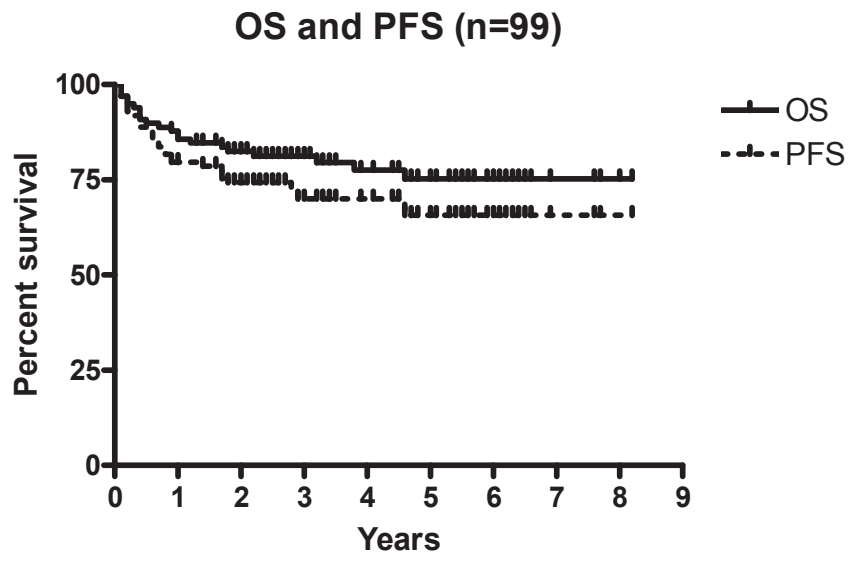

Figure 2. The probability of overall survival and progression- free survival in 99 patients treated with an anthracycline- based regimen and rituximab.

the diagnosis of DLBCL and to distinguish GC-like and non-GC-like immunophenotype groups according to the algorithm of Hans et al. [5] (Fig. 1a, 1b) Haematoxylin-eosin stained slides served for the selection of representative blocks for immunohistochemical and molecular genetic analysis. Commercially available monoclonal antibodies CD20 (L26, DAKO, 1:50), CD3 (SP7, Neomarkers, 1:50), CD10 (56C6, Novocastra, 1:100), bcl-6 (PG-B6p, DAKO, 1:20) and IRF4/ MUM1 (MUM1p, DAKO, 1:50) were used in the indirect immunoperoxidase methods with N-Histofine Simple Stain MAX PO (Nichirei Biosciences) with diaminobenzidine to distinguish the two immunophenotypes. Heat-induced epitope retrieval was performed in $10 \mathrm{mM}$ sodium citrate buffer pH 6.0 (CD20), Target Retrieval Solution DAKO (CD3), $1 \mathrm{mM}$ EDTA pH 8.0 (CD10), Target Retrieval Solution pH 9.0 DAKO (bcl-6) and Target Retrieval Solution High pH DAKO
(IRF4/MUM1) in a water bath during a 40 minute period at $98^{\circ} \mathrm{C}$. Room temperature for 50 minutes was used in all primary antibody incubations with the only one exception of anti-CD10 antibody incubated overnight at $4^{\circ} \mathrm{C}$.

Statistical evaluation. Survival was compared according to the characteristics of overall survival and progression free survival. Overall survival (OS) was characterised as the time from diagnosis to the last follow-up or death from any cause. Progression free survival (PFS) was defined as the time from diagnosis to an event (disease progression or death from any cause) or to the last follow-up. Survival probability was determined using the Kaplan Meier method and the differences were calculated using the long-rank test. The Graph Pad and SPSS software were used for these calculations.

\section{Results}

In the set of 120 patients immunohistochemically examined total of 52 patients were evaluated as GC type and 68 patients as having non-GC. Correlation with clinical characteristics and survival analysis was possible in the sample of 99 patients, the GC type was found in 45 and non-GC in 54 cases in this subset of patients (Tab. 1). The median age was 61 years (22-83); 52 were men. Twenty eight patients had performance status ECOG 2 and more. B symptoms were present in 42 patients. Sixty one patients had higher LDH values, 15 patients had more than 1 extranodal site involved. Advanced clinical stages were present in 64 patients and 35 patients had an intermediate or high IPI. There was found no statistical difference between GC and nonGC group.

With a median follow-up of 3.13 years the probability of OS and PFS at 3 years was $81 \%$ (95\% CI 72.9-88.9) and 70\% (95\% CI 60.7- 79.5), respectively. At five years, OS and PFS were $75.1 \%$ (95\% CI 72.9-79.5) and 65.7\% (95\% CI 55.0-76.3), respectively-see Fig. 2.

Table 1. The clinical parameters of the evaluated set

\begin{tabular}{|c|c|c|c|c|c|c|c|c|}
\hline \multicolumn{2}{|l|}{ Patients } & \multicolumn{2}{|c|}{ whole sample } & \multicolumn{2}{|c|}{ GC } & \multicolumn{2}{|c|}{ Non-GC } & $\mathrm{p}$ \\
\hline \multicolumn{2}{|l|}{$\mathrm{n}$} & \multicolumn{2}{|c|}{99} & \multicolumn{2}{|c|}{45} & \multicolumn{2}{|c|}{54} & ns \\
\hline \multicolumn{2}{|c|}{ age (median) } & \multicolumn{2}{|c|}{$22-83(61)$} & \multicolumn{2}{|c|}{$22-74(57)$} & \multicolumn{2}{|c|}{$22-83(61)$} & ns \\
\hline \multicolumn{2}{|c|}{ male/female } & \multicolumn{2}{|c|}{$51 / 48$} & \multicolumn{2}{|c|}{$22 / 23$} & \multicolumn{2}{|c|}{$29 / 25$} & ns \\
\hline \multicolumn{2}{|l|}{$\mathrm{CS} \mathrm{I}+\mathrm{II}$} & 32 & $33.3 \%$ & 14 & $31.8 \%$ & 18 & $34.6 \%$ & ns \\
\hline \multicolumn{2}{|c|}{ CS III+IV } & 64 & $66.7 \%$ & 30 & $68.2 \%$ & 34 & $65.4 \%$ & ns \\
\hline \multicolumn{2}{|c|}{ LDH above normal range } & 61 & $64.2 \%$ & 25 & $61.0 \%$ & 36 & $66.7 \%$ & ns \\
\hline \multicolumn{2}{|c|}{ PS 2 and higher } & 28 & $29.2 \%$ & 14 & $32.6 \%$ & 14 & $26.4 \%$ & ns \\
\hline \multirow[t]{4}{*}{ IPI } & low & 27 & $30.0 \%$ & 14 & $34.1 \%$ & 13 & $26.5 \%$ & ns \\
\hline & low-intermediate & 27 & $30.0 \%$ & 13 & $31.7 \%$ & 14 & $28.6 \%$ & ns \\
\hline & high -intermediate & 17 & $18.9 \%$ & 5 & $12.2 \%$ & 12 & $24.5 \%$ & ns \\
\hline & high & 19 & $21.1 \%$ & 9 & $22.0 \%$ & 10 & $20.4 \%$ & $\mathrm{~ns}$ \\
\hline \multirow[t]{4}{*}{ therapy } & CHOP like & 87 & $87.9 \%$ & 37 & $82.2 \%$ & 50 & $92.6 \%$ & ns \\
\hline & intensive CHOP & 12 & $12.1 \%$ & 8 & $17.8 \%$ & 4 & $7.4 \%$ & ns \\
\hline & ASCT & 12 & $12.1 \%$ & 6 & $13.3 \%$ & 6 & $11.1 \%$ & ns \\
\hline & rituximab & 99 & $100.0 \%$ & 45 & $100.0 \%$ & 54 & $100.0 \%$ & ns \\
\hline
\end{tabular}

NB: The following parameters were evaluated in the corresponding number of patients: CS 96 pts, LDH 95 pts, PS 96 pts, IPI 90 pts 
OS: $G C$ versus non $G C(n=99)$

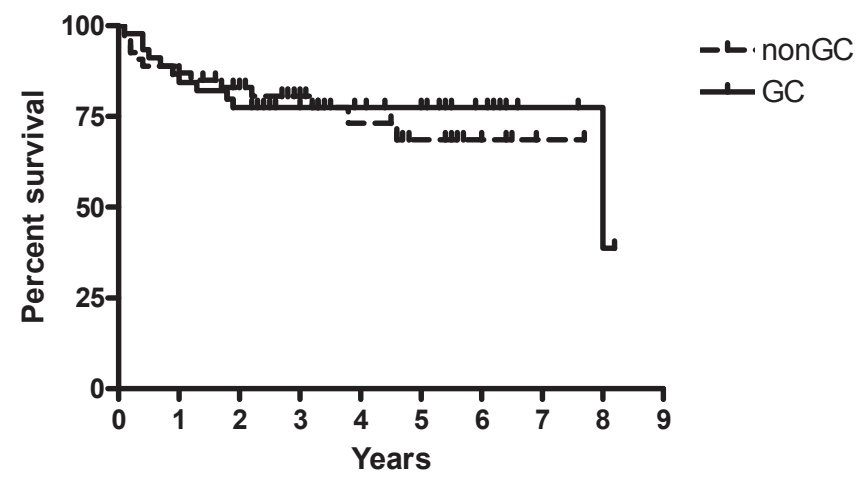

Figure 3. Overall survival in 99 patients with DLBCL treated with an anthracycline regimen- comparison of the GC and non-GC group according to the Hans algorithm.

Using the Hans algorithm, we categorized 45 patients into the GC group and 54 patients into the non-GC group. There was no statistically significant difference in the individual clinical characteristics between the two groups.

Table 1 shows an overview of the clinical characteristics of both groups.

Evaluation of the clinical response. Response rate was comparable in both groups (Tab. 2). In the GC group, the overall response rate (ORR) was $90.7 \%$, with $76 \%$ of patients achieving complete remission and $7 \%$ of patients achieving partial remission. In the non-GC group, the ORR was $89.8 \%$ with $75.5 \%$ of patients achieving complete remission and $8.2 \%$ of patients achieving partial remission.

Survival analysis. OS and PFS at 3 years was $81 \%$ (95\% CI 72.9-88.9) and 70\% (95\% CI 60.7- 79.5), respectively and at 5 years $75.1 \%$ (95\% CI 72.9-79.5) and 65.7\% (95\% CI 55.076.3), respectively. Neither the median OS nor median PFS were reached.

Clinical Prognostic Factors. The predictive role of individual clinical characteristics on survival was studied- age, CS, LDH, PS, IPI, as well as the type of DLBCL according to IHC were tested in univariate analysis. The following factors were found to have significant impact on PFS and OS respectively:

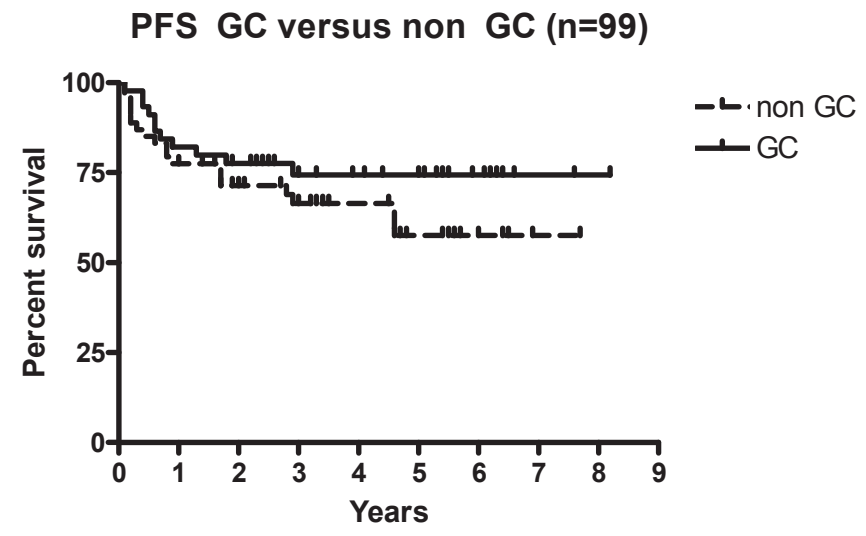

Figure 4. PFS in 99 patients with DLBCL treated with an anthracycline regimen- comparison of the GC and non-GC group according to the Hans algorithm.

age ( $\mathrm{p}=0.001$ and 0.0011 resp.), $\mathrm{LDH}(\mathrm{p}=0.061$ and 0.005 resp.), performance status ( $\mathrm{p}=0.02$ and 0.04$)$ and IPI $(\mathrm{p}<0.0001$ and 0.0001 resp.).

Comparing both groups of DLBCL subtypes using univariate analysis, we found no significant difference between both evaluated groups in OS ( $\mathrm{p}=0.405)$ (Fig.3) nor in PFS $(\mathrm{p}=0.219)$ (Fig. 4).

Two multivariate (MV) analyses were performed: one for the age, clinical stage, LDH, PS, type of DLBCL according to IHC and second one for IPI and type of DLBCL according to IHC. In the first multivariate analysis, factors that independently influenced PFS and OS respectively were age $(p<0.0001$ and $<0.0001$ resp.), $\mathrm{LDH}$ ( $\mathrm{p}=0.30$ and $\mathrm{p}=0.05$ resp.) and clinical stage ( $\mathrm{p}=0.02$ and 0.04 resp.). Subtypes according to the IHC evaluation did not have impact on either PFS or OS in multivariate analysis.

The IPI was significantly associated with OS $(\mathrm{p}<0.0001)$ and PFS $(\mathrm{p}<0.0001)$, when assessed by the Cox model.

\section{Discussion}

Determining DLBCL subtypes using molecular genetic methods (gene expression analysis) in the era of rituximab

Table 2. Response rate to chemotherapy

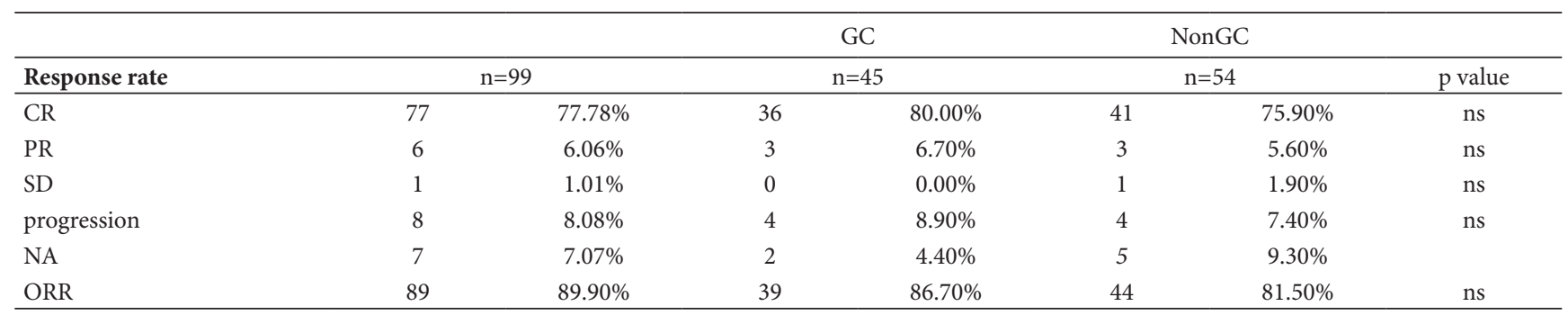

NA - not applicable 
has a prognostic value [8]. The discrimination of different subtypes has, however, not only prognostic impact, but based on the different pathogenesis and different pathways it can be used as the tool for targeted therapy. Several papers have been published demonstrating the different efficacy of bortezomib in combination with chemotherapy in patients with ABC vs. GCB lymphoma [9]. Similarly, the efficacy of lenalidomide in monotherapy has been demonstrated as significantly different in nonGC vs. GC subtype [10]. GEP however still requires the availability of fresh (frozen) material and is rather expensive. Hans et al [5] published in 2004 the first widely accepted and used algorithm for dividing DLBCL into the GC type and nonGC subtype. This algorithm was based on the immunohistochemical expression of $\mathrm{CD}$ 10 , bcl- 6 and MUM 1 proteins. Over the years, a number of papers have been published, some of which confirm the prognostic division according to Hans $[8,10-13]$ while others failed to find statistically significant differences between these two groups [14-18].

Other immunohistochemical algorithms attempt to improve the predictive significance of this method using the antibodies GCET 1, CD10, bcl-6, MUM 1, FOXP1 [19], bcl 2, CD 10, MUM 1 [20], FOXP1, MUM 1 [8] and LMO2 [21] expression. Newly proposed the Tally algorithm [11] tries to avoid sequential assessment of antibodies used in previous schemes. Its results are comparable to previous algorithms. Its main disadvantage is using of five antibodies, because in the widely accepted Hans algorithm only three commercially easily accessible and widely used antibodies are required.

In our set of patients, immunohistochemical classification of DLBCL using the most frequently applied Hans algorithm did not demonstrate any significant differences neither for PFS nor OS between the subtype classified as GC and that one as non-GC. The only prognostic marker confirmed in our cohort of patients was the International prognostic index IPI.

Our observations are in concordance with published results, having failed to confirm the significance of classifying DLBCL subtypes according to Hans. It is thus also in concordance with recently published works [14,22]. Our study involves almost one hundred patients treated with immuno-chemotherapy and we have demonstrated the applicability and utility of clinical prognostic markers used to date, namely IPI but we failed to confirm the prognostic impact of the Hans immunohistochemical algorithm.

According to literature, concordance of the Hans method with gene expression profiling (GEP) is $70-80 \%[5,23]$ with the remaining $20-30 \%$ cases being discrepant. It cannot be ruled out that the reason for the different results in our study might be the imprecise stratification of our sample. Meyer et al [11] showed that although the individual immunohistochemical algorithms may be capable to demonstrate a significant difference between the GC and non-GC subtypes, in each algorithm these subtypes consist of different patients. In other words, GC lymphoma according to one algorithm can be classified as non-GC lymphoma according to another algorithm. An- other possible reason may be the issue of determining Bcl-6 positivity, whereby discrepancies in technical performance in different laboratories and the interpretation of samples between individual pathologists have been described in literature $[8,24]$. Nevertheless, if the algorithms using Bcl-6 (according to Hans or Choi) were modified by its exclusion, they gave similar results as unmodified ones [11].

We cannot, however, exclude the possibility, that Hans algorithm correctly discriminates GC and nonGC subtypes in our cohort, but there is no different PFS and OS outcome between these subtypes in our cohort. It has been shown that the predictive value may be suppressed by more intensive chemotherapy regimens and eventually by autologous transplant [16]. We do not consider this could be the explanation for similar outcome of GC and non-GC subtype in our series, because only $12 \%$ of all patients received intensive treatment and transplantation and the proportion was similar in each subgroup.

It thus appears that using immunohistochemical algorithms, in our case the Hans one, can be confounding for identifying different subtypes with different prognosis to test intensified therapy or therapeutic modalities targeting different pathogenetic mechanisms in the GC and non-GC subtypes. As GEP still remains unavailable in most cases of DLBCL, it is necessary to look for other means to discriminate between GC -like and nonGC-like DLBCL. The immunohistochemistry is still a favourite method due to its reasonable costs, the reproducibility of the results have to be however improved.

\section{Conclusion}

The DLBCL consists of at least two subtypes GCB and $\mathrm{ABC}$ (nonGCB) which can be distinguished by GEP. The suggested more practical immunohistochemistry algorithms are not however reproducible in all studied cohorts. We demonstrate that the most frequently used Hans algorithm failed to discriminate GC and nonGC in terms of different survival probability in our cohort treated with immunochemotherapy.

Acknowledgements: This work was supported by Ministry of Health, Czech Republic [Grant NS9791-4/2008].

\section{References}

[1] SWERDLOW S, CAMPO E, HARRIS N, JAFFE E, PILERI S, et al. World Health Organization Classification of Tumours of Haematopoietic and Lymphoid Tissues Lyon 2008: IARC Press. 439 p.

[2] TRNENY M, VASOVA I, PYTLIK R, BELADA D, JANKOVSKA M, et al. The Non-Hodgkind's Lymphoma subtypes distribution and survival in Czech Republic. Klin Onkol 2007; 20: 340-348.

[3] ALIZADEH AA, EISEN MB, DAVIS RE, MA C, LOSSOS IS, et al. Distinct types of diffuse large B-cell lymphoma identi- 
fied by gene expression profiling. Nature 2000; 403: 503-511. http://dx.doi.org/10.1038/35000501

[4] LENZ G, WRIGHT G, DAVE SS, XIAO W, POWELL J, et al. Stromal gene signatures in large-B-cell lymphomas. N Engl J Med 2008; 359: 2313-2323. http://dx.doi.org/10.1056/ NEJMoa0802885

[5] HANS CP, WEISENBURGER DD, GREINER TC, GASCOYNE RD, DELABIE J, et al. Confirmation of the molecular classification of diffuse large B-cell lymphoma by immunohistochemistry using a tissue microarray. Blood 2004; 103: 275-282. http://dx.doi.org/10.1182/blood-2003-05-1545

[6] CHESON BD, HORNING SJ, COIFFIER B, SHIPP MA, FISHER RI, et al. Report of an international workshop to standardize response criteria for non-Hodgkin's lymphomas. NCI Sponsored International Working Group. J Clin Oncol 1999; 17: 1244.

[7] TRNENY M, PYTLIK R, BELADA D, KUBACKOVA K, VASOVA I, et al. Treatment of Diffuse Large B-Cell Lymphoma with Rituximab, Intensive Induction and High-Dose Consolidation: The Final Analysis of the Czech Lymphoma Study Group (CLSG) R-MegaCHOP-ESHAP-BEAM (R-MEB) Trial. ASH Annual Meeting Abstracts 2007; 110: 21.

[8] NYMAN H, JERKEMAN M, KARJALAINEN-LINDSBERG ML, BANHAM AH, LEPPA S Prognostic impact of activated B-cell focused classification in diffuse large B-cell lymphoma patients treated with R-CHOP. Mod Pathol 2009; 22: 10941101. http://dx.doi.org/10.1038/modpathol.2009.73

[9] DUNLEAVY K, PITTALUGA S, CZUCZMAN MS, DAVE SS, WRIGHT G, et al. Differential efficacy of bortezomib plus chemotherapy within molecular subtypes of diffuse large B-cell lymphoma. Blood 2009; 113: 6069-6076. http://dx.doi. org/10.1182/blood-2009-01-199679

[10] HERNANDEZ-ILIZALITURRI FJ, DEEB G, ZINZANI PL, PILERI SA, MALIK F, et al. Higher response to lenalidomide in relapsed/refractory diffuse large B-cell lymphoma in nongerminal center B-cell-like than in germinal center B-celllike phenotype. Cancer 2011; 117: 5058-5066. http://dx.doi. org/10.1002/cncr.26135

[11] MEYER PN, FU K, GREINER TC, SMITH LM, DELABIE J, et al. Immunohistochemical methods for predicting cell of origin and survival in patients with diffuse large B-cell lymphoma treated with rituximab. J Clin Oncol 2011; 29: 200-207. http://dx.doi.org/10.1200/JCO.2010.30.0368

[12] CHEN Z, DU Z, CHEN J, BAO Y, TANG F Prognostic evaluation of immunohistochemical profiles in diffuse large B-cell lymphoma: a Chinese study. Med Oncol 2011; 28: 241-248. http://dx.doi.org/10.1007/s12032-010-9433-3

[13] FANG C, XU W, LI JY A systematic review and meta-analysis of rituximab-based immunochemotherapy for subtypes of diffuse large B cell lymphoma. Ann Hematol 2010; 89: 1107-1113. http://dx.doi.org/10.1007/s00277-010-0990-5

[14] CASTILLO JJ, BELTRAN BE, SONG MK, ILIC I, LEPPA S, et al. The Hans algorithm is not prognostic in patients with diffuse large B-cell lymphoma treated with R-CHOP. Leuk Res 2012. http://dx.doi.org/10.1016/j.leukres.2011.12.012

[15] MORTON LM, CERHAN JR, HARTGE P, VASEF MA, NEPPALLI VT, et al. Immunostaining to identify molecular subtypes of diffuse large B-cell lymphoma in a populationbased epidemiologic study in the pre-rituximab era. Int J Mol Epidemiol Genet 2011; 2: 245-252.

[16] GU K, WEISENBURGER DD, FU K, CHAN WC, GREINER TC, et al. Cell of origin fails to predict survival in patients with diffuse large B-cell lymphoma treated with autologous hematopoietic stem cell transplantation. Hematol Oncol 2011.

[17] OH S, KOO DH, SUH C, KIM S, PARK BH, et al. Prognostic value of immunohistochemical biomarkers at different cut-off values in patients with diffuse large B-cell lymphoma treated with CHOP chemotherapy. J Korean Med Sci 2011; 26: 1556-1562. http://dx.doi.org/10.3346/ jkms.2011.26.12.1556

[18] JABLONSKA J, JESIONEK-KUPNICKA D, POTEMSKI P, KOWALIK A, SYGUT J, et al. Comparison of two different immunohistochemical algorithms identifying prognostic subgroups of DLBCL. Pol J Pathol 2010; 61: 124-132.

[19] CHOI WW, WEISENBURGER DD, GREINER TC, PIRIS MA, BANHAM AH, et al. A new immunostain algorithm classifies diffuse large B-cell lymphoma into molecular subtypes with high accuracy. Clin Cancer Res 2009; 15: 5494-5502. http://dx.doi.org/10.1158/1078-0432.CCR-09-0113

[20] MURIS JJ, MEIJER CJ, VOS W, VAN KRIEKEN JH, JIWA NM, et al. Immunohistochemical profiling based on Bcl-2, CD10 and MUM1 expression improves risk stratification in patients with primary nodal diffuse large B cell lymphoma. J Pathol 2006; 208: 714-723. http://dx.doi.org/10.1002/path.1924

[21] NATKUNAM Y, FARINHA P, HSI ED, HANS CP, TIBSHIRANI R, et al. LMO2 protein expression predicts survival in patients with diffuse large B-cell lymphoma treated with anthracycline-based chemotherapy with and without rituximab. J Clin Oncol 2008; 26: 447-454. http://dx.doi.org/10.1200/ LCO.2007.13.0690

[22] HONG J, PARK S, PARK J, KIM HS, KIM KH, et al. Evaluation of prognostic values of clinical and histopathologic characteristics in diffuse large B-cell lymphoma treated with rituximab, cyclophosphamide, doxorubicin, vincristine, and prednisolone therapy. Leuk Lymphoma 2011; 52: 1904-1912. http://dx.doi.org/10.3109/10428194.2011.588761

[23] VEELKEN H, VIK DANNHEIM S, SCHULTE MOENTING J, MARTENS UM, FINKE J, et al. Immunophenotype as prognostic factor for diffuse large B-cell lymphoma in patients undergoing clinical risk-adapted therapy. Ann Oncol 2007; 18: 931-939. http://dx.doi.org/10.1093/annonc/mdm012

[24] WINTER JN, WELLER EA, HORNING SJ, KRAJEWSKA M, VARIAKOJIS D, et al. Prognostic significance of Bcl-6 protein expression in DLBCL treated with CHOP or R-CHOP: a prospective correlative study. Blood 2006; 107: 4207-4213. http://dx.doi.org/10.1182/blood-2005-10-4222 